\title{
Digital Image Enhancement in Matlab: An Overview on Histogram Equalization and Specification
}

\author{
Rezoana Bente Arif ${ }^{*}$, Mohammad Mahmudur Rahman Khan ${ }^{2 \#}$, and Md. Abu Bakr Siddique ${ }^{1 @}$ \\ ${ }^{1}$ Dept. of EEE, International University of Business Agriculture and Technology, Dhaka 1230, Bangladesh \\ ${ }^{2}$ Dept. of ECE, Mississippi State University, Mississippi State, MS 39762, USA \\ rezoana@iubat.edu*,mrk303@msstate.edu*, absiddique@iubat.edu@ \\ Corresponding Author: absiddique@iubat.edu ${ }^{\circledR}$
}

\begin{abstract}
This paper has two major parts. In the first part histogram equalization for the image enhancement was implemented without using the built-in function in MATLAB. Here, at first, a color image of a rat was chosen and the image was transformed into a grayscale image. After this conversion, histogram equalization was implemented on the grayscale image. Later on, in the same image for each RGB channel, histogram equalization was implemented to observe the effect of histogram equalization on each channel. In the end, the histogram equalization was implemented to this specific color image of a rat. In the second part, for the grayscale image in part 1 , the desired histogram of another colored image of a rat was introduced and histogram specification was implemented on the original colored image.
\end{abstract}

Keywords-Digital image processing, color image, grayscale image, histogram equalization, histogram specification, image enhancement, RGB channel

\section{INTRODUCTION}

In the modern age, image enhancement technique becomes a vital tool to facilitate with the improvement in image quality in various sectors like identifying anything in images as well as medical imaging [1, 2], computational photography, forensic analysis [3], and pattern recognition [4] in machine vision applications. Main motive of this technique is to make the images discernible by correcting the color hue and brightness imbalance [5] as well as contrast adjustment [6]. As the background of an image may hide structural information of an image [7], the technique to prolong the image temperament, enhances the foreground information, while retaining the background information and thus increases the overall contrast of an image $[8,9]$. Several algorithmic techniques such as Artificial Neural Network [10], Convolutional neural Network [11], and Knearest Neighbors [12] can also be applied in image processing techniques such as segmentation, thresholding and filtering. Though there are several image enhancement techniques has been developed over the past decades, the histogram based image enhancement techniques specifically; 1) Histogram equalization, 2) Histogram specification are utilized vastly for their high efficiency and simplicity of algorithm [5].

The counterbalance process of Histogram improves the contrast of an image through the propagation of effectively spreading out the most uniform values throughout the histogram of an image [13]. As the active spreading of intensity values makes the dark pixels appear darker and the white pixels lighter, the histogram equalization improves the visual appearance of an image. In short, histogram equalization corresponds to the uniform distribution of a histogram [14]. Histogram equalization can be applied both in grayscale as well as RGB color images for contrast enhancement.

The histogram specification is the alteration of an image so that its histogram matches a particular desired histogram [15]. In the histogram specification, cumulative frequency distributions (CDFs) are calculated from the histogram of an image to adjust the shape of the source image's histogram to be suited the expected image's histogram. Histogram specification is a handy technique for image contrast enrichment and thus improving visual image quality.

In this paper, histogram equalization and specification techniques are implemented for image enhancement. At first, a color image of a mouse is transmuted to the grayscale image. Then, histogram equalization is applied to the grayscale image. Histogram equalization is also implemented on red channel, green channel and blue channel separately and lastly, combined them to find histogram equalized color image. Lastly, histogram specification is also performed on the same color image given a desired image's histogram.

\section{LITERATURE REVIEW}

Histogram equalization has been showing its vast contribution in improvement of image quality in numerous sectors like medical images [16], pattern imaging [4] and so on. Previously, the performances of different types of histogram equalization such as; Global Histogram Equalization, Local Histogram Equalization and Fast Quadratic Dynamic Histogram Equalization in peak signal to noise ratio was performed [17]. To improve the contrast of images of MRI (Magnetic resonance imaging) tests for sensitive treatments (e.g. cancer, soft tissue, stroke etc.) the performances of four types of histogram equalizations were granted [16]. Though the quality in enhanced color images are no better than the gray scale enhanced image quality, approaches were made with 3-D Color Histogram Equalization method [18]. Recently, cohesion of 1-d and 2D Histogram based method was analyzed in researches [19]. Again, another version of histogram called histogram of compressed scattering coefficients (HCSC) was proposed to get salvation from low-level hand-crafted features in images [20]. Conversely, instead of the generosity in histogram enhancement and specification method, there are a bunch of drawbacks in this method to improve the contrast level in the images. Due to inappropriate contrast, proper images are often not detected in X-rays or lung cancer etc [21]. Two of 
the image enhancement methods; Histogram equalization (HE) as well as Contrast Limited Adaptive Histogram Equalization (CLAHE) left unrealistic images after enhancement in embedded system [22]. Our proposal regarding Histogram Equalization and Specification method provides a uniform response in contrast improvement which gives an appropriate image in the output. On top of that, analyze was performed by MATLAB coding without any built-in function.

\section{III.HISTOGRAM EQUALIZATION AND SPECIFICATION BASICS}

Like any other unsupervised methods such as Fuzzy CMeans [23] and ADBSCAN [24] clustering, histogram equalization is also unsupervised. The histogram of a digital image with $L$ total possible intensity levels in the range [0, $\mathrm{G}]$ is defined as the detached function:

$$
h\left(r_{k}\right)=n_{k} \ldots \ldots(1)
$$

The value of $\mathrm{G}$ is 255 for images of category uint8. Often, it is useful to work with normalized histogram as follows:

$$
p\left(r_{k}\right)=\frac{n_{k}}{n}
$$

The normalized range of the predominance level is $[0,1]$. In the probability density function, difference of the PDFs of the input and output images are denoted by the subscript. The following metamorphosis on the input levels is performed to attain output (processed) intensity levels, s,

$$
s=T(r)=\int_{0}^{r} p_{r}(w) d w
$$

Here,

$p_{r}(r)=$ The probability density function (PDF) of the severity levels in the original image the intensity.

An image is produced by the former transformation that contains similar intensity levels. On top of that it transcends the whole range $[0,1]$. The genuine outcome of this equalization process for intensity level is an image which contains onward progressive range as well as better contrast. The transformation function is nothing over than the cumulative distribution function (CDF).

For discrete quantities the equalization transformation becomes:

$$
S_{k}=\sum_{j=1}^{k} \frac{n_{j}}{n} \ldots \ldots
$$

An adjustable transformation function is generated by histogram equalization in the histogram equalization process of the original image. Though the transformation function for an image is flexible, it does not get changed once it is calculated. Specification of the shape of the histogram is necessary to get the processed image for some distinctive implementations. Histogram specification is the process in which an image having a particular histogram is brought forth.
$H(z)=\int_{0}^{z} p_{z}(w) d w=s$

$\mathrm{s}$ could be found from the input image (this is the histogramequalization transformation), so it follows that the following equation could be used to detect the altered levels $\mathrm{z}$ whose PDF is the specified, $p_{z}(z)[15,25]$.

$z=H^{-1}(s)=H^{-1}[T(r)]$

\section{IV.IMPLEMENTATION AND DISCUSSION OF RESULTS}

The following image was used to implement histogram equalization and specification.

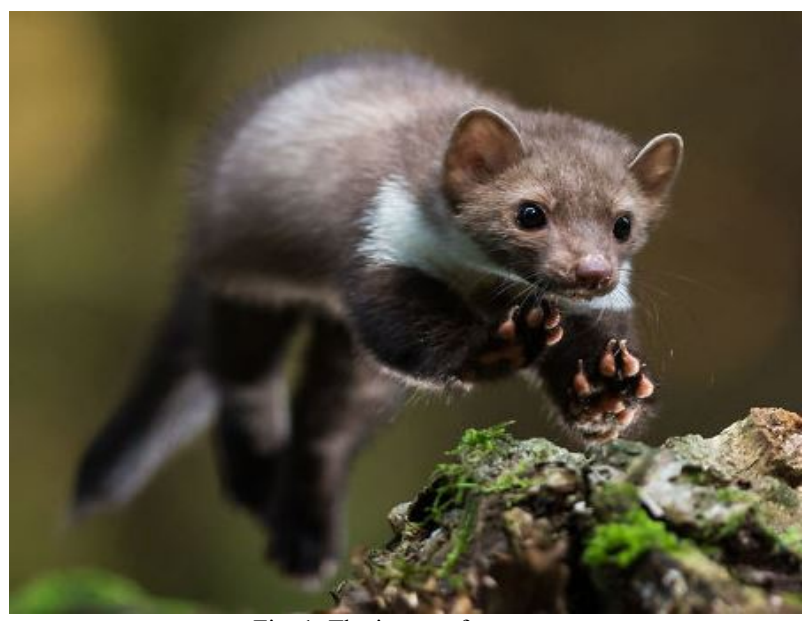

Fig. 1. The image of a mouse

In Figure 2 the resolved grayscale image is represented after the conversion to the grayscale image.

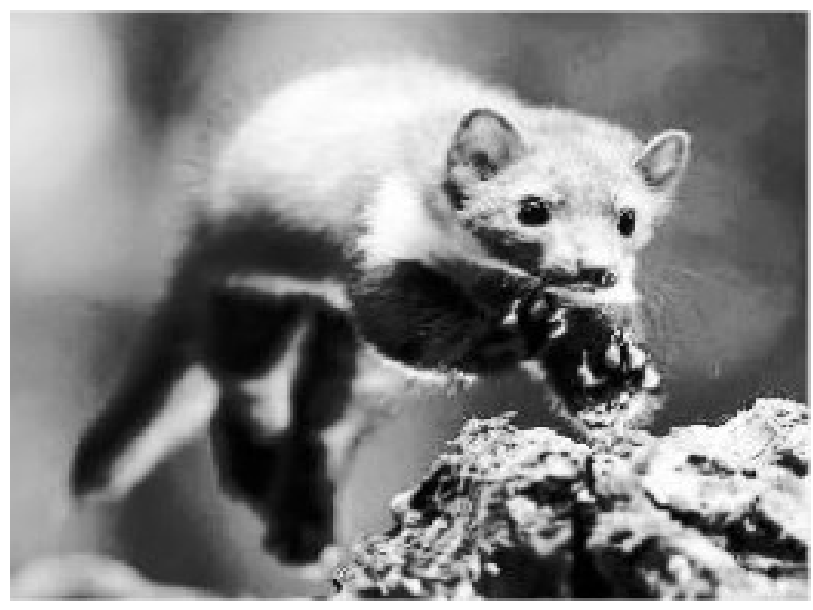

Fig. 2. The grayscale image of figure 1

Then histogram equalization was implemented on the image without using the built-in function in MATLAB. After the implementation the obtain histogram equalized image is made known in figure 3 along with the genuine grayscale image. The histograms of the genuine grayscale image in addition to histogram equalized image are also shown. 

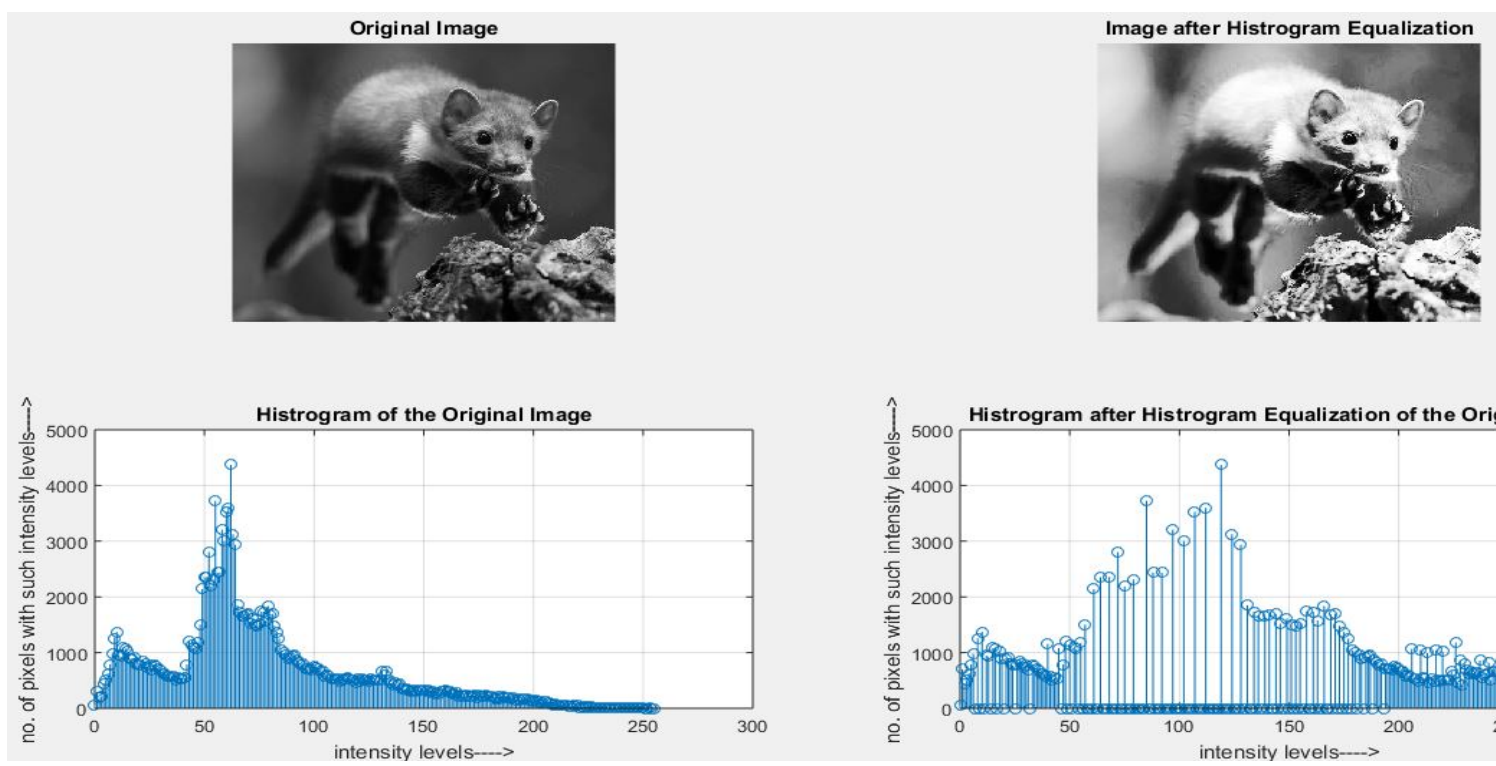

Fig. 3. The original grayscale rat image along with image after histogram equalization with their respective histograms

From the figure above, it is evident that the original image has most of the pixel clustered in a specific range of intensity. However, after histogram equalization, the intensity spread throughout the total intensity range which causes the raise in the sharpness of the image.

Afterwards, 3 RGB channels were prepared in the original image, and for each channel, the histogram equalization was applied.
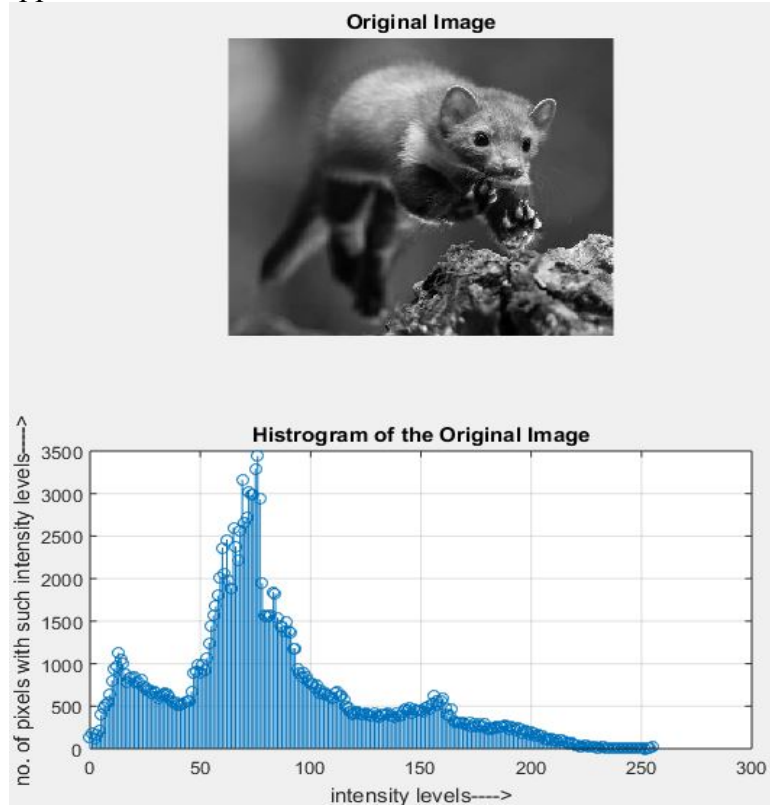

Fig. 4. The original red channel image along with image after histogram equalization with their respective histograms

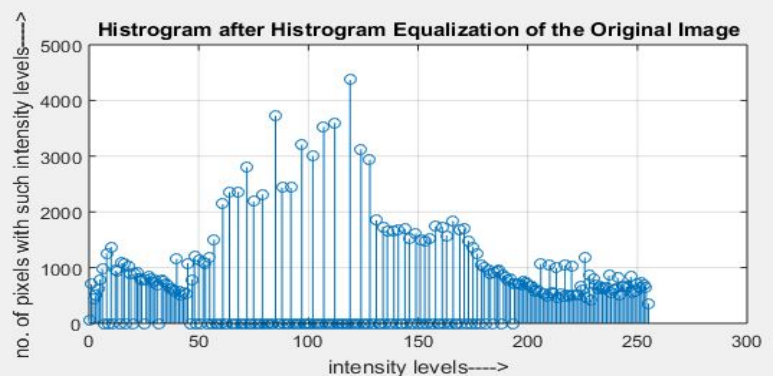

Here, Figure 4, Figure 5 and Figure 6 demonstrate the obtained images for three different channels named Red, green and blue channel respectively after the standardization by histogram. The histograms of original RGB channels grayscale images, in addition to histogram after histogram equalization of RGB channel images, are also shown.
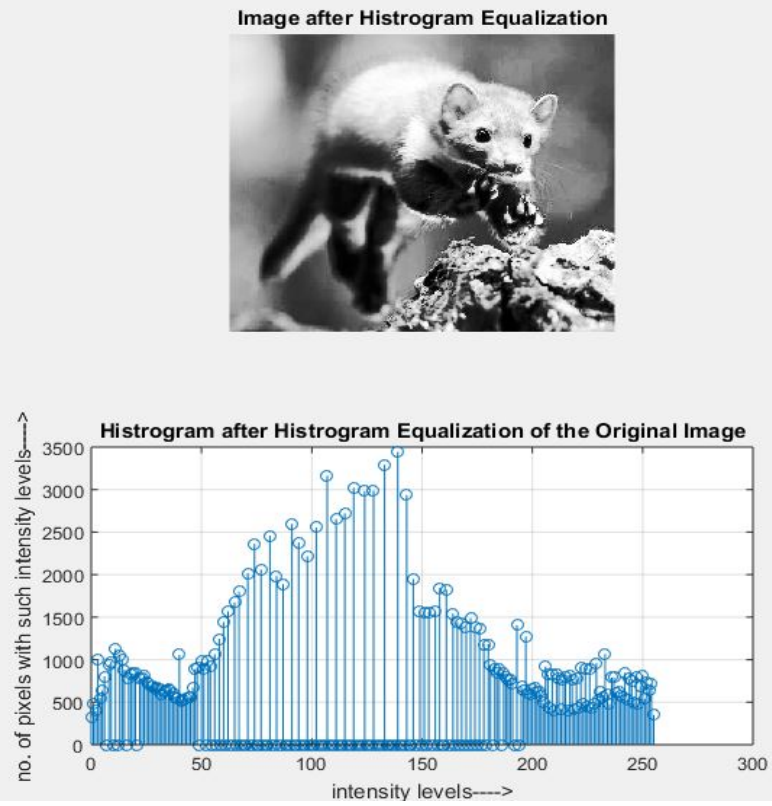


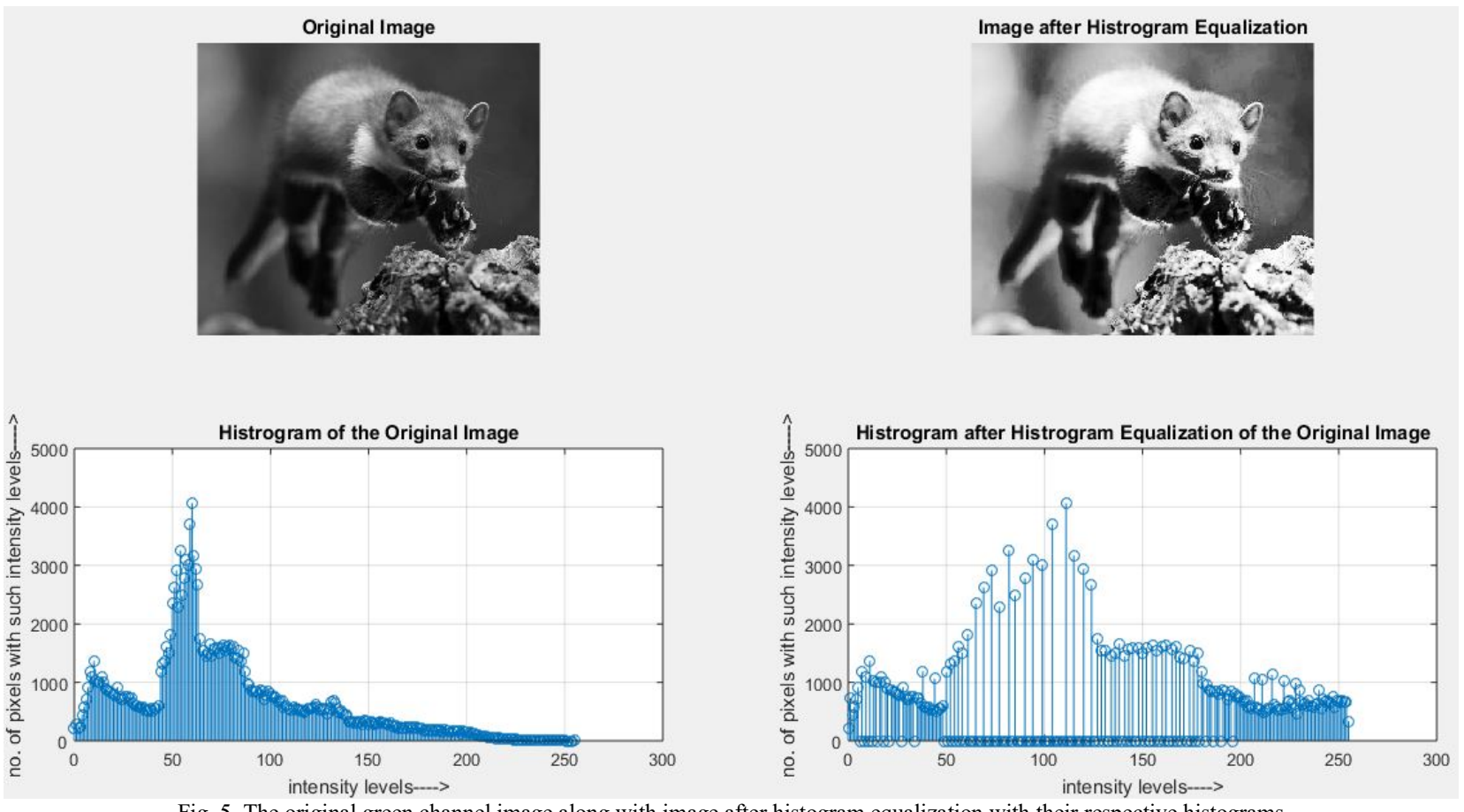

Fig. 5. The original green channel image along with image after histogram equalization with their respective histograms
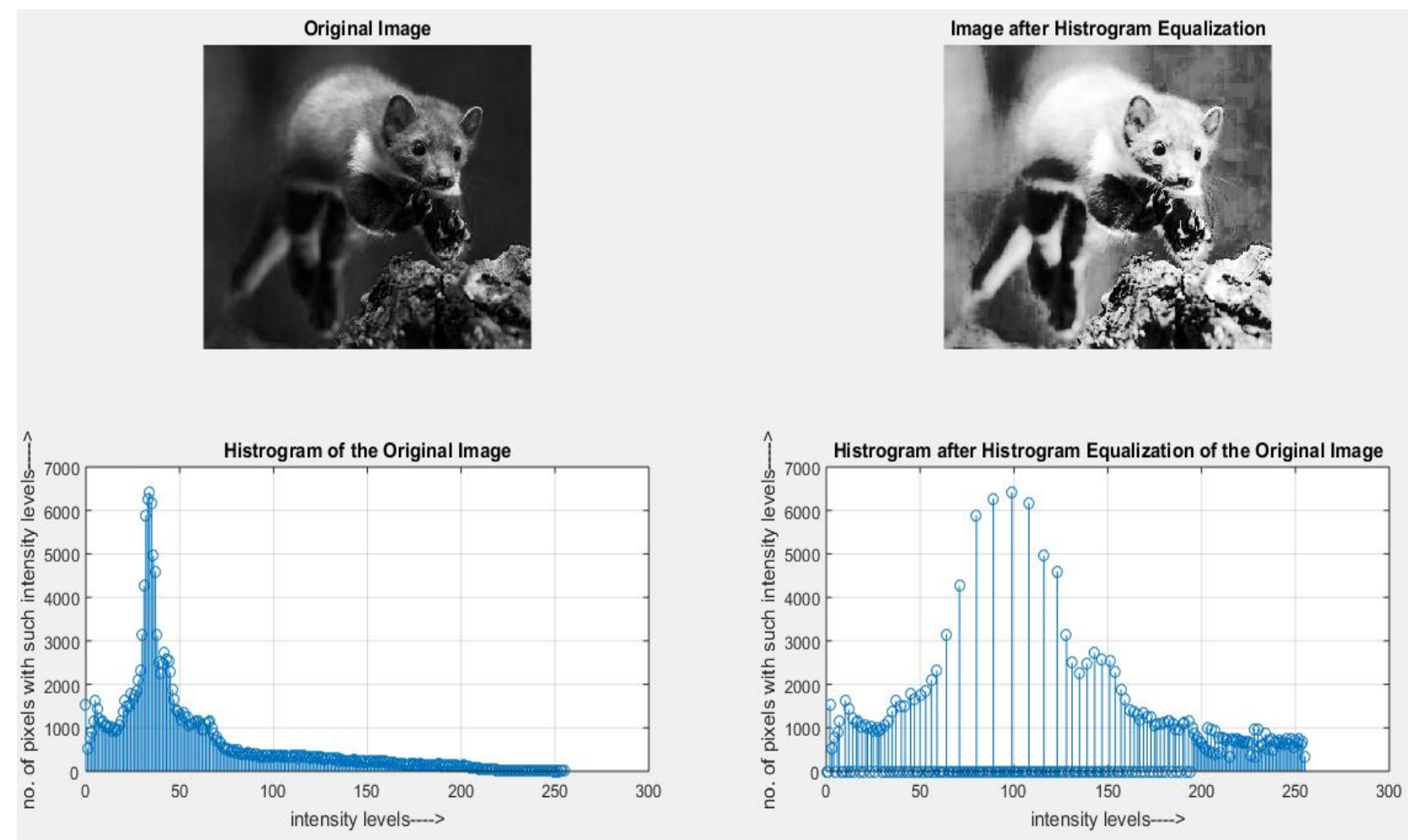

Fig. 6. The original blue channel image along with image after histogram equalization with their respective histograms

Here, it is detected that each channel shows different contrast enhancement patterns. Then the three channels were added, and the equalization process was implemented for the real color image.
By doing so, the obtain image after histogram equalization is depicted in figure 7 along with the original color image. The histograms of original color image, and the histograms of histogram equalized image, are also shown. 


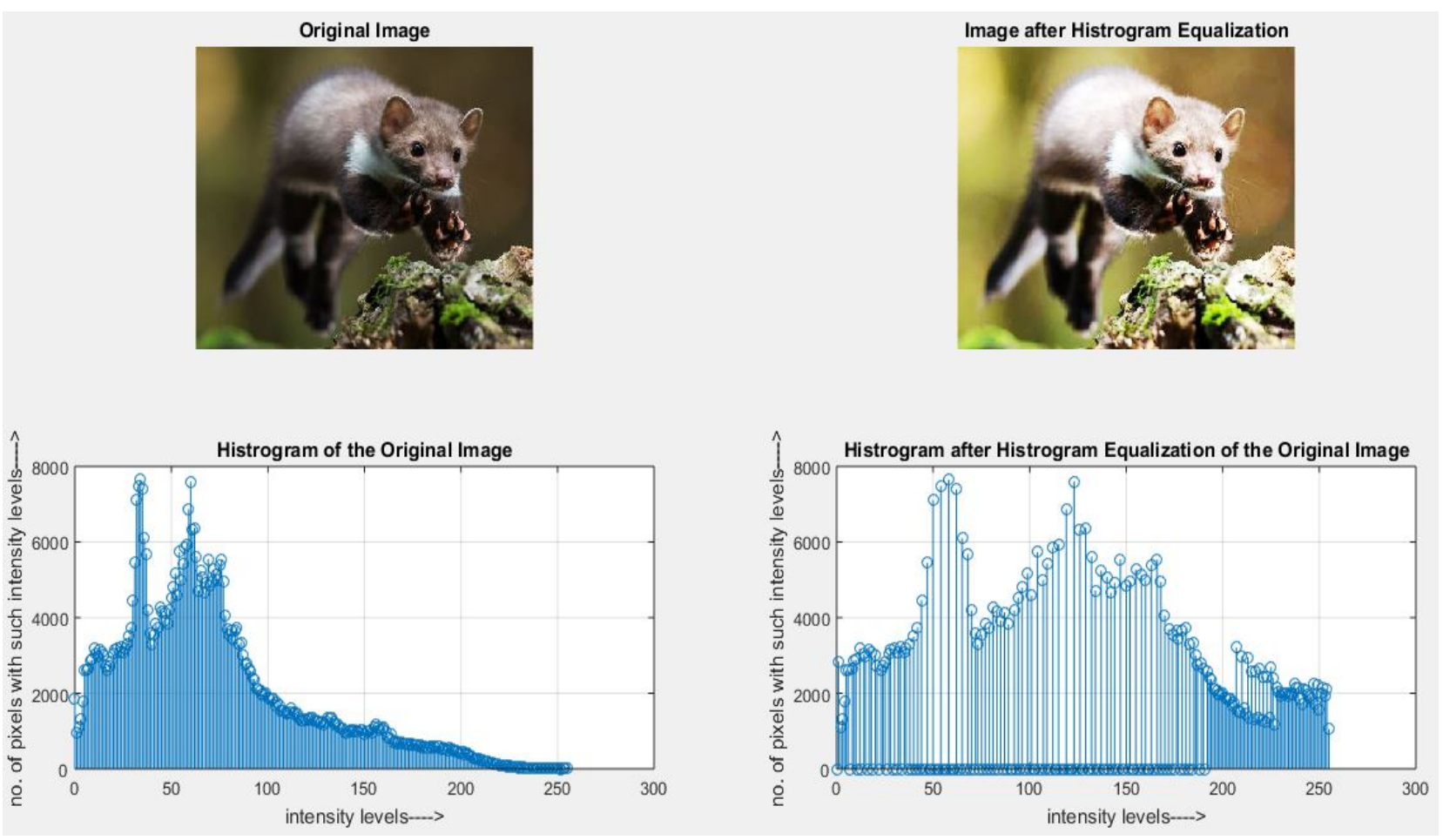

Fig. 7. The original color image along with image after histogram equalization with their respective histograms

In the figure above, the original image is shadier than the histogram equalized image.

For histogram specification, the image in Figure 8 was considered to extract the desired histogram for the image in Figure 1.

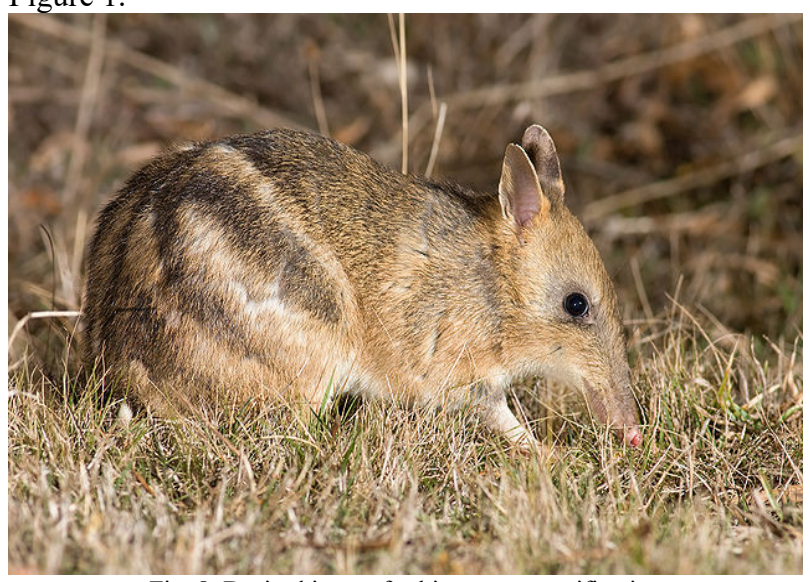

Fig. 8. Desired image for histogram specification
At first, the histograms of both of the images (original and desired images) were extracted. Then the histogram equalization was applied to the original image and the histogram of the original image was matched to the desired histogram through histogram specification process. Finally, the output of the histogram specification is publicized in Figure 9.

Here, it is noticed that at first, the images had histograms of a different pattern. However, after the histogram specification, the histogram of the actual image took a similar appearance to the specified image histogram. 

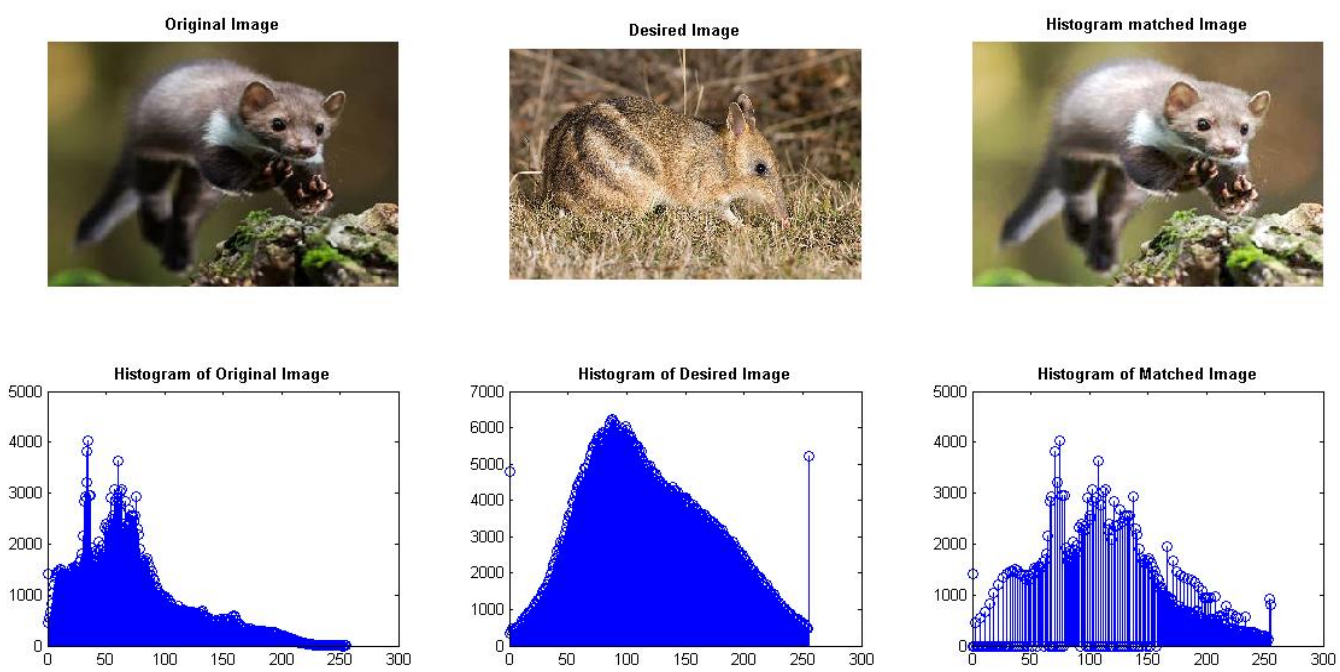

Fig. 9. The original color image along with desired image and image after histogram specification with their respective histograms

\section{CONCLUSION}

This paper presents equalization and specification of histogram techniques' practical implementation on some images for image contrast enhancement. It is observed that these techniques work quite well to improve pictures visual quality. In future works of this series, some other improved image enhancement techniques will be discussed.

\section{REFERENCES}

[1] T.-L. Ji, et al., "Adaptive image contrast enhancement based on human visual properties," IEEE transactions on medical imaging, vol. 13, pp. 573-586, 1994.

[2] Y. Yang, et al., "Medical image enhancement algorithm based on wavelet transform," Electronics letters, vol. 46, pp. 120-121, 2010.

[3] X. Yu, et al., "Contrast enhanced subsurface fingerprint detection using high-speed optical coherence tomography," IEEE Photonics Technol. Lett., vol. 29, pp. 70-73, 2017.

[4] H. Kuang, et al., "Combining region-of-interest extraction and image enhancement for nighttime vehicle detection," IEEE Intelligent Systems, vol. 31, pp. 57-65, 2016.

[5] B. Xiao, et al., "Brightness and contrast controllable image enhancement based on histogram specification," Neurocomputing, vol. 275, pp. 2798-2809, 2018.

[6] E. Peli, "Contrast in complex images," JOSA A, vol. 7, pp. 2032-2040, 1990.

[7] J. Fu, et al., "Wavelet-based histogram equalization enhancement of gastric sonogram images," Computerized medical imaging and graphics, vol. 24, pp. 59-68, 2000.

[8] J. Tang, et al., "Image enhancement using a contrast measure in the compressed domain," IEEE Signal Processing Letters, vol. 10, pp. 289-292, 2003.

[9] S. DelMarco and S. Agaian, "The design of wavelets for image enhancement and target detection," in Mobile Multimedia/Image Processing, Security, and Applications 2009, 2009, p. 735103.

[10] Md. Abu Bakr Siddique, et al., "Study and Observation of the Variations of Accuracies for Handwritten Digits Recognition with Various Hidden Layers and Epochs using Neural Network Algorithm," in 2018 4th International Conference on Electrical Engineering and Information \& Communication Technology (iCEEiCT), 2018, pp. 118123.

[11] Rezoana Bente Arif, et al., "Study and Observation of the Variations of Accuracies for Handwritten Digits Recognition with Various Hidden Layers and Epochs using Convolutional Neural Network," arXiv preprint arXiv: $1809.06187,2018$.
[12] Mohammad Mahmudur Rahman Khan, et al., "Study and Observation of the Variation of Accuracies of KNN, SVM, LMNN, ENN Algorithms on Eleven Different Datasets from UCI Machine Learning Repository," in 2018 4th International Conference on Electrical Engineering and Information \& Communication Technology (iCEEiCT), 2018, pp. 124-129.

[13] S.-D. Chen, "A new image quality measure for assessment of histogram equalization-based contrast enhancement techniques," Digital Signal Processing, vol. 22, pp. 640-647, 2012.

[14]B. Fittes, "Gray-level transformations for interactive image enhancement. MS Thesis. Final Technical Report," 1975.

[15]R. C. Gonzalez and R. E. Woods, "Digital image processing," ed: Prentice hall New Jersey, 2002.

[16]H. Kaur and J. Rani, "MRI brain image enhancement using Histogram equalization Techniques," in Wireless Communications, Signal Processing and Networking (WiSPNET), International Conference on, 2016, pp. 770-773.

[17] S. Patel and M. Goswami, "Comparative analysis of Histogram Equalization techniques," in Contemporary Computing and Informatics (IC3I), 2014 International Conference on, 2014, pp. 167168.

[18] J.-H. Han, et al., "A novel 3-D color histogram equalization method with uniform 1-D gray scale histogram," IEEE Transactions on Image Processing, vol. 20, pp. 506-512, 2011.

[19] D. Kim and C. Kim, "Contrast Enhancement Using Combined 1-D and 2-D Histogram-Based Techniques," IEEE Signal Processing Letters, vol. 24, pp. 804-808, 2017.

[20]R. Lan and Y. Zhou, "Medical image retrieval via histogram of compressed scattering coefficients," IEEE journal of biomedical and health informatics, vol. 21, pp. 1338-1346, 2017.

[21]S. Avinash, et al., "Analysis and comparison of image enhancement techniques for the prediction of lung cancer," in Recent Trends in Electronics, Information \& Communication Technology (RTEICT), 2017 2nd IEEE International Conference on, 2017, pp. 1535-1539.

[22]M. Sahani, et al., "Design of an embedded system with modified contrast limited adaptive histogram equalization technique for realtime image enhancement," in Communications and Signal Processing (ICCSP), 2015 International Conference on, 2015, pp. 0332-0335.

[23] M. Siddique, et al., "Implementation of Fuzzy C-Means and Possibilistic C-Means Clustering Algorithms, Cluster Tendency Analysis and Cluster Validation," arXiv preprint arXiv:1809.08417, 2018.

[24] Mohammad Mahmudur Rahman Khan, et al., "ADBSCAN: Adaptive Density-Based Spatial Clustering of Applications with Noise for Identifying Clusters with Varying Densities," in 2018 4th International Conference on Electrical Engineering and Information \& Communication Technology (iCEEiCT), 2018, pp. 107-111. 